\title{
Development of an Antimicrobial Blended White LED System Containing Pulsed 405-nm LEDs for Decontamination Applications.
}

\author{
Jonathan B. Gillespie ${ }^{\mathrm{a}}$, Michelle Maclean ${ }^{\mathrm{a}, \mathrm{b}}$, Mark P. Wilson ${ }^{\mathrm{a}}$, Martin J. Given ${ }^{\mathrm{a}}$, Scott J. \\ MacGregor $^{\mathrm{a}}$ \\ ${ }^{\mathrm{a}}$ Department of Electronic \& Electrical Engineering, University of Strathclyde, 204 George St, \\ Glasgow, G11XW, UK; ${ }^{b}$ Department of Biomedical Engineering, University of Strathclyde, 106 \\ Rottenrow East, Glasgow, G4 0NW, UK.
}

\begin{abstract}
This study details the design, build and testing of a prototype antimicrobial blended white light unit containing pulsed red, yellow, green and $405 \mathrm{~nm}$ LEDs. With a push for alternative methods of disinfection, optical methods have become a topic of interest. Ultra-violet (UV) light is widely known for its antimicrobial properties however; $405 \mathrm{~nm}$ light has demonstrated significant antimicrobial properties against many common hospital acquired pathogens. In this study, a pulsed, blended, white-light prototype with a high content of $405 \mathrm{~nm}$ antimicrobial light, was designed, built and tested. Antimicrobial efficacy testing of the prototype was conducted using Staphylococcus aureus and Pseudomonas. aeruginosa, two bacteria which are common causes of hospital acquired infections. These were exposure to 3 different light outputs from the prototype and the surviving bacteria enumerated. Results showed that the mixed light output provided a much better CRI and light output under which to work. Also, the light output containing $405 \mathrm{~nm}$ light provided an antimicrobial effect, with decontamination of $10^{3} \mathrm{CFUml}^{-1}$ populations of both bacterial species. The other light content (red, yellow, green) had no beneficial or adverse effects on the antimicrobial properties of the $405 \mathrm{~nm}$ light. The results suggest that with further development, it could be possible to produce an antimicrobial blended white light containing pulsed $405 \mathrm{~nm}$ light that could supplement or even replace standard white lighting in certain environments.
\end{abstract}

Keywords: 405nm light, Pulsed LEDs, blending, disinfection, environmental decontamination, antimicrobial, violet light, efficacy

\section{INTRODUCTION}

Infection control is an issue of key importance in healthcare. Antibiotic resistance is on the rise and has become somewhat of a driving force for alternate methods of disinfection technology. This study focuses on a specific method of optical disinfection through the use of violet-blue light in the region of $400420 \mathrm{~nm}$, with particular efficacy in the region of $405 \mathrm{~nm}$. $405 \mathrm{~nm}$ light has been shown to possess significant antimicrobial properties ${ }^{1-6}$ and is being developed towards clinical applications, with a major focus on its potential use for continuous environmental decontamination of occupied environments ${ }^{7,8}$. UV-light has been widely researched and used and is highly effective for the inactivation of bacteria ${ }^{9}$, however, its high energy means that it can also be harmful to humans. $405 \mathrm{~nm}$ light sits just above UV-light in the visible light region of the electromagnetic spectrum, which means it can be used at levels that are safe for human exposure, whilst still being lethal to bacteria ${ }^{10-12}$.

Previous research has demonstrated that pulsed operation of $405 \mathrm{~nm}$ light emitting diodes (LEDs) can provide more efficient disinfection, both in terms of electrical energy consumption and inactivation achieved per unit of optical energy expended $^{13}$. A consideration when using $405 \mathrm{~nm}$ light for environmental disinfection is that $405 \mathrm{~nm}$ light alone can be uncomfortable to work under due to its violet-blue colour and poor colour rendering index (CRI), and in under-lit conditions, the $405 \mathrm{~nm}$ light can be hard to work under. Also due to shorter wavelength violet and blue light scattering more than longer wavelength yellow and red light (known as Rayleigh Scattering) it can be difficult to focus the eye at this wavelength region ${ }^{14}$. Additionally, under $405 \mathrm{~nm}$ light alone, white materials glows super-bright, akin to the effects of so called 'black light'/long-wave UV-A light.

Coupled with the recent findings that pulsed $405 \mathrm{~nm}$ LEDs can provide improved electrical and operational efficiency, it is of interest to investigate the potential for pulsed LEDs to be blended to provide a white light which would have 
antimicrobial properties whilst being aesthetically acceptable for use for continuous environmental disinfection in occupied environments. The present study details the design, build and antimicrobial efficacy testing of a small-scale prototype system employing pulsed 405nm, red, yellow and green LEDs controlled with Pulse Width Modulation (PWM) via a microcontroller.

\section{SYSTEM DESIGN}

As mentioned, one of the main issues with using $405 \mathrm{~nm}$ light in occupied environments is that $405 \mathrm{~nm}$ light alone provides a very poor CRI under which to work, and also results in a phosphorescence-like glow from white objects. Ideally, the amount of $405 \mathrm{~nm}$ light in a system that would provide additional room lighting should be reduced to a point where the antimicrobial effects are still at a practical level, and it should be balanced by other wavelengths of lights to aid colour blending and improve the visual aesthetics.

Previous studies have been carried out with $405 \mathrm{~nm}$ lighting designs incorporating varieties of cool and warm white LEDs in combination with the $405 \mathrm{~nm}$ light arrays ${ }^{7,8}$ however there are difficulties when trying to balance very intense $405 \mathrm{~nm}$ light with white light which already contains a significant blue content.

The core idea of the system design in the present study was to use pulsed $405 \mathrm{~nm}$ LEDs alongside pulsed red, yellow and green LEDs in order to create a blended antimicrobial white light source. The LEDs and the pulsing circuit used to control the pulsing LED setup are shown in Table 1 and Figure 1, respectively. Pulsing was used to control the blending of the $405 \mathrm{~nm}$, red, yellow and green light; specifically the duty cycle was altered to control the average intensity of each colour.

Table 1: Details of the light-emitting diodes (LEDs) used in the prototype blended pulsed LED system

\begin{tabular}{|l|l|c|l|}
\hline Light-Emitting Diode & Make/Model & Number used & $\begin{array}{l}\text { Peak Wavelength } \\
\text { /FWHM }\end{array}$ \\
\hline $405 \mathrm{~nm}$ & Nichia NCSU276AT-U405 & 4 & $405 \mathrm{~nm} / 12 \mathrm{~nm}$ \\
Green & Lumileds LXML-PM01-0090 & 2 & $530 \mathrm{~nm} / 30 \mathrm{~nm}$ \\
Yellow & Lumileds LXML-PL01-0060 & 2 & $590 \mathrm{~nm} / 28 \mathrm{~nm}$ \\
Red & Lumileds LXM2-PH01-0070 & 2 & $617 \mathrm{~nm} / 28 \mathrm{~nm}$ \\
\hline
\end{tabular}

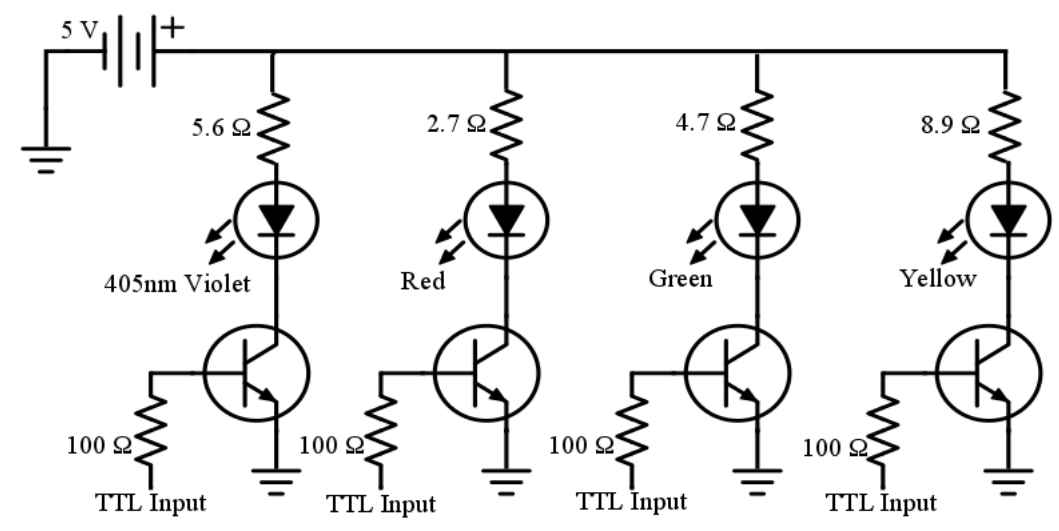

Figure 1: Circuit diagram of the pulsing circuit used to control the blended pulsed LED system.

Each of the four colours of LEDs was powered from the same power source, an AC-DC converter (Traco Power TXM 075-105) which converted mains power (230VAC) to 5VDC. Each colour of LED was operated at a different voltage 
and current, thus a different value of resistor was used for each branch of the pulsing circuitry to ensure control of the voltage dropped across the LEDs. In addition, each LED in the circuit (Figure 1) represents an array of LEDs in parallel due to a single LED not being capable of producing enough light for the current application. with the system used $4 \times$ $405 \mathrm{~nm}$ LEDs, $2 \times$ Red LEDs, $2 \times$ Yellow LEDs, and $2 \times$ Green LEDs. This consequently meant that each parallel branch containing an LED also contained a single resistor of the corresponding value, given in Figure 1. Each of the transistors (STMicroelectronics BD239C) was controlled by an MSP430 microcontroller, which coordinated electrical pulses to switch on and off the transistors and thus the LEDs. The microcontroller allowed for fast pulsing, and it was decided that for this study and setup, the pulse frequency would be $1 \mathrm{kHz}$ based on the experiments carried out in previous work by Gillespie et $a l^{13}$. To create a blend of light, duty cycles of $66 \%, 66 \%, 80 \%$ and $50 \%$ were used for the red, yellow, green and 405nm LEDs, respectively. These specific percentages were chosen by visual and spectral assessment in an attempt to achieve a white blend. Additionally, the percentages were quantized in steps due to the way the code was written on the microcontroller although not to a point were it became an issue.

The LEDs were mounted, with thermal adhesive, on an aluminum plate that acted as a heat sink. This was then mounted inside a polyvinyl chloride (PVC) housing unit with a single $200 \mathrm{~mm}$ diameter circular aperture. Due to this being a small-scale proof-of-concept system, the optical output intensity was low therefore a diffuser for improved blending was not included in the design at this stage. A photograph of the prototype is shown in Figure 2.

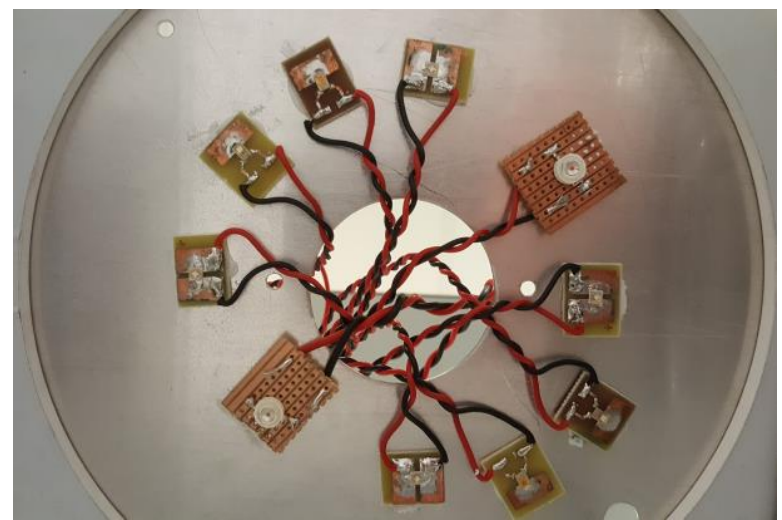

Figure 2: A close-up view of the arrangement of the individual LEDs mounted on the aluminum heat sink as seen from the underside through the $200 \mathrm{~mm}$ aperture.

The spectral output of the system compared with a standard warm white LED (Lumileds, LXML-PWW1-0060), measured using a spectrometer (Ocean Optics HR4000) and SpectraSuite software, is shown in Fig 3.

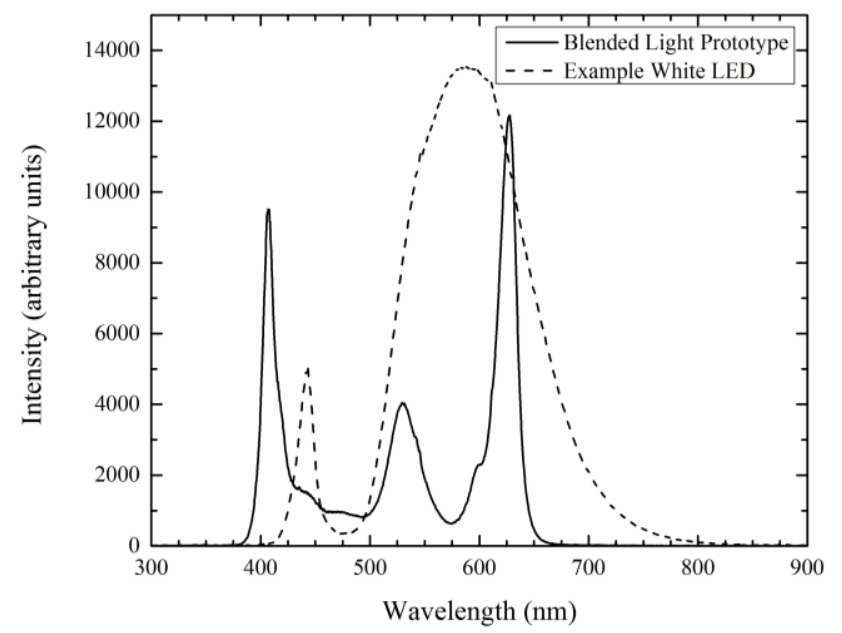

Figure 3: Comparison of the output spectrum of the prototype blended pulsed unit and a standard white LED, measured using Ocean Optics HR4000 spectrometer and SpectraSuite software. 


\section{MATERIALS, METHODS \& EXPERIMENTAL DESCRIPTION}

\subsection{Bacterial Preparation}

Both bacteria to be used in the experiments, Staphylococcus aureus NCTC 4135 (National Collection of Type Cultures, Colindale, UK) and Pseudomonas aeruginosa NCTC 9009, were cultured at $37^{\circ} \mathrm{C}$ for $18 \mathrm{hrs}$ in $100 \mathrm{ml}$ nutrient broth (Oxoid Ltd, UK), under rotary conditions (120 rpm). Each culture was then centrifuged at $3939 \times g$ for 10 minutes at $20^{\circ} \mathrm{C}$, re-suspended in $100 \mathrm{ml}$ phosphate buffered saline, and serially diluted to a density of $10^{3}$ colony forming units per millilitre $\left(\mathrm{CFUml}^{-1}\right)$ for experimental use.

\subsection{Exposure of Bacterial Samples using the Pulsed LED System}

For all experiments, $100 \mu \mathrm{l}$ of the $10^{3} \mathrm{CFUml}^{-1}$ bacterial suspensions were pipetted and spread onto $50 \mathrm{~mm}$ nutrient agar (Oxoid Ltd., UK) plates. This sample volume provided a population density of approximately $250 \mathrm{CFU} / \mathrm{plate}$, and was selected as a good representation of the typical levels of contamination that can be collected from occupied environments using contact plate sampling ${ }^{8}$. These plates were positioned at a distance of $10 \mathrm{~cm}$ below the pulsed LED system and exposed one of the three light outputs detailed below. Identical control samples were prepared which were exposed to normal laboratory lighting for the same durations. Post-exposure, all sample plates were incubated at $37^{\circ} \mathrm{C}$ for 24 hours, and surviving populations were then enumerated.

Three different lighting outputs were used for exposure of bacterial samples. The microcontroller coding was altered in each case to switch different colours on and off, however all other parameters were kept the same (frequency, duty cycle and intensity). The 3 different light outputs were: (1) all LEDs (red, yellow, green and 405nm) on, referred to as $R Y G$ 405 mix; (2) the 405nm LEDs alone, with red, yellow and green LEDs switched off, referred to as 405 light alone; and (3) red, yellow and green LEDs switched on and $405 \mathrm{~nm}$ LEDs switched off, referred to as RYG mix.

Bacterial sample plates were exposed for up to 60 minutes, in 15-minute intervals, for each of the 3 different light outputs. Experiments were carried out in triplicate, with 3 sample plates exposed each time.

The optical emission spectrum of the 3 light mixes was measured and the combined spectrum is shown in Figure 4 . Irradiance was measured directly below the light source at a distance of $10 \mathrm{~cm}$ (at the positions of the sample plates) using a photodiode detector (Model-1Z02413; Ophir) and radiant optical power meter (Model-70260; Oriel Instruments) calibrated at $405 \mathrm{~nm}$. The average irradiance of the 405nm light LEDs, when operated in both the 405 light alone and $R Y G-405$ mix was measured to be $1.45 \mathrm{mWcm}^{-2}$. Due to the power meter having a response time in the region of 0.1 seconds, and the pulsing frequency being $\approx 1 \mathrm{kHz}$, the power meter was not capable of measuring the peak intensity therefore it is the average irradiance readings that have been used in the present study. With this and the knowledge that the $405 \mathrm{~nm}$ LEDs are being pulsed at a 50\% duty cycle, it can be deduced that the peak intensity of the $405 \mathrm{~nm}$ light is double that of the average measured by the power meter, $\approx 2.9 \mathrm{mWcm}^{-2}$. The power meter was used to ensure the light output of the RYG-405 mix and the RYG mix was consistent.

\subsection{Data Analysis}

The experimental data are reported as \% surviving bacterial population, compared to the equivalent control population, and represent the mean values of a minimum of triplicate independent experimental results ( $\geq 3$ ). Standard Deviation (SD) was calculated and used for all data and graphical representation. Data-sets were analysed using one-way ANOVA and Tukey post-hoc test with OriginPro 9.0.0 statistical software, with significant differences accepted at $\mathrm{P} \leq 0.05$.

\section{EXPERIMENTAL RESULTS}

\subsection{Development of the Blended Pulsed White Light}

Due to this being an early proof-of-concept study, the light output and resulting CRI were the main objectives in terms of system design. This system included no diffuser or light mask thus the LEDs were completely visible and the different colours discernable when looking directly at the light source, however when assessing the optical output emitting onto 
the surface below the prototype, it was apparent that the blend of light from the pulsed circuit LED system was more comparable to a blended white light. An illustration of the visual improvement in the light output between the $405 \mathrm{~nm}$ light alone and the $R Y G-405$ mix is shown in Fig 5.
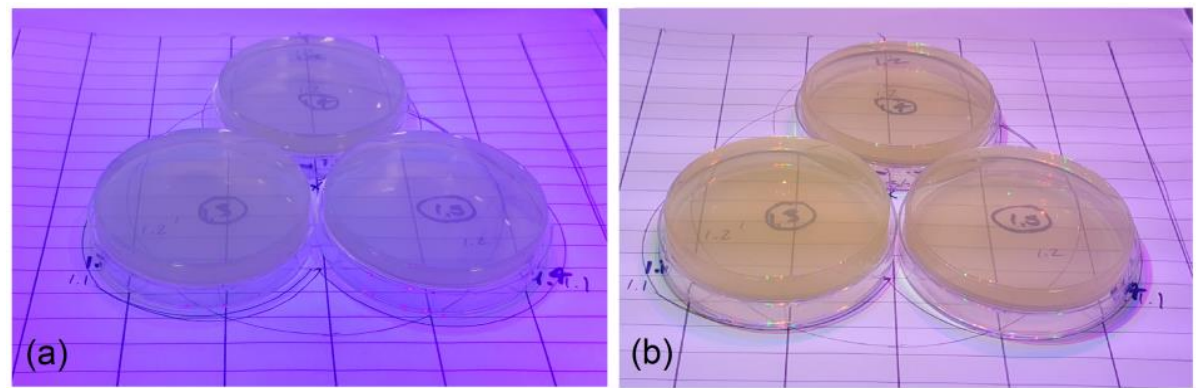

Figure 4: Comparison of the visual appearance of agar sample plates when illuminated under (a) 405nm light alone and (b) the $R Y G-405$ mix.

Figure 5 demonstrates the difference the added colour makes in terms of the CRI under the light. It was much easier to perceive the natural colour of the agar plates under the $R Y G-405 \mathrm{mix}$. It still has a purple tinge to it, however the demonstration and prototype show that it should be possible to achieve a blend comparable to white light with further development and a higher content of the other 3 colours to create a better spectral spread closer to a white light spectrum.

\subsection{Pulsed 405nm Light Alone Exposure}

One of the major aspects in the design of this prototype pulsed system was to ensure that a sufficient level of $405 \mathrm{~nm}$ light was included to enable evaluation of its antimicrobial efficacy. To assess this, the first step was to setup the prototype using the 405nm light LEDs alone. S. aureus and $P$. aeruginosa seeded sample plates were exposed to the $405 \mathrm{~nm}$ light for increasing increments of time as detailed in Section 3.2, and the results are shown in Figure 5 below.

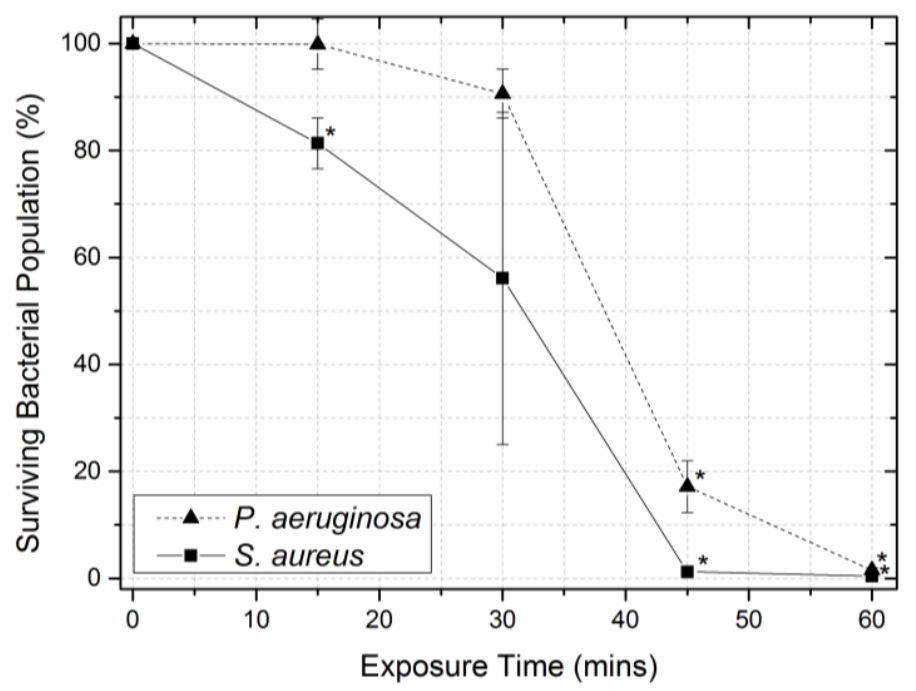

Figure 5: Inactivation of $S$. aureus and $P$. aeruginosa by exposure to the pulsed $405 \mathrm{~nm}$ light alone. Surviving bacterial populations are calculated as a percentage of the equivalent non-exposed control population $( \pm \mathrm{SD}) . *$ denotes a statistically significant decrease in population following light exposure $(\mathrm{p} \leq 0.05)$.

Exposure to pulsed $405 \mathrm{~nm}$ alone induced a $3-\log _{10}$ reduction in both bacterial populations. Due to the large standard deviation for the $S$. aureus 30-minute exposure time, significant inactivation (compared to the non-exposed control populations) was not evidenced until 45 minutes exposure, as was the case with $P$. aeruginosa. 


\subsection{Pulsed RYG-405 mix Light Exposure}

The prototype was setup to provide output of red, yellow, green and $405 \mathrm{~nm}$ light, and sample plates seeded with $S$. aureus and $P$. aeruginosa were exposed to the light for increasing increments of time as detailed in Section 3.2. The results of this exposure are shown in Figure 6.

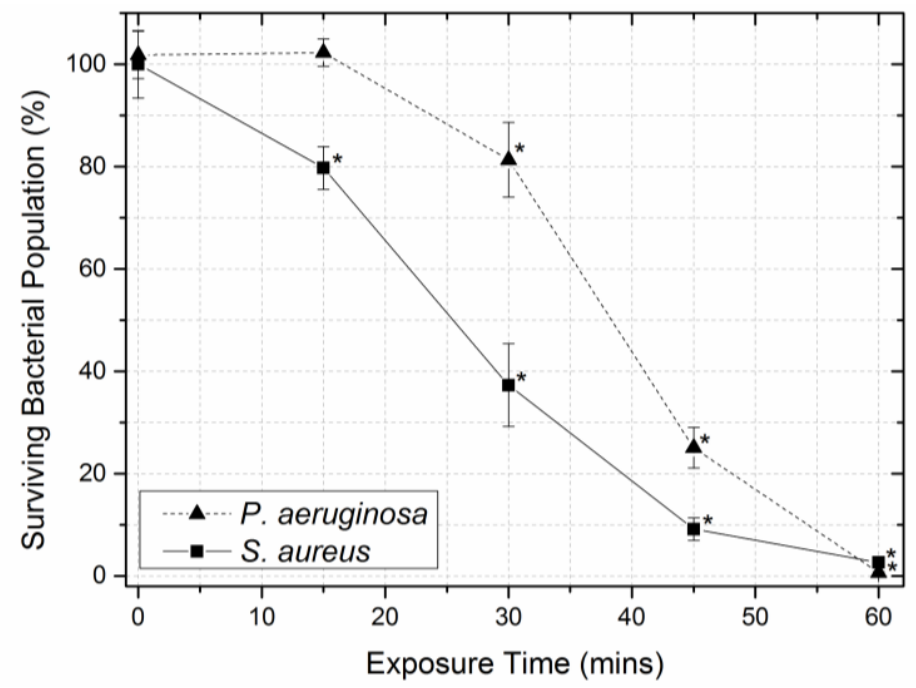

Figure 6: Inactivation of $S$. aureus and $P$. aeruginosa by exposure to the pulsed $R Y G-405$ mix. Surviving bacterial populations are calculated as a percentage of the equivalent non-exposed control population $( \pm \mathrm{SD}) . *$ denotes a statistically significant decrease in population following light exposure $(\mathrm{p} \leq 0.05)$.

As was the case with the $405 \mathrm{~nm}$ light alone, the results show a downward trend with approximately a 3- $\log _{10}$ reduction in bacterial population achieved for both strains of bacteria. Significant inactivation was demonstrated after 15 minute exposure for $S$. aureus and 30 minute exposure for $P$. aeruginosa. The corresponding controls for both inactivation curves hold steady at $10^{3} \mathrm{CFUml}^{-1}$ for the full exposure time. These results show that even with low intensities of $405 \mathrm{~nm}$ light blended with the 3 other colours, populations of $S$. aureus and P. aeruginosa, representative of the densities present in the environment, can be successfully inactivated.

\subsection{Pulsed RYG mix Light Exposure}

As a final test, the prototype was setup to operate without the $405 \mathrm{~nm}$ content to establish whether the other three colours provide any significant contribution to the antimicrobial operation. The system was set-up to provide pulsed output of red, yellow and green light, and bacterial were exposed for the same time increments as in the previous experiments. The results are shown in Figure 7. 


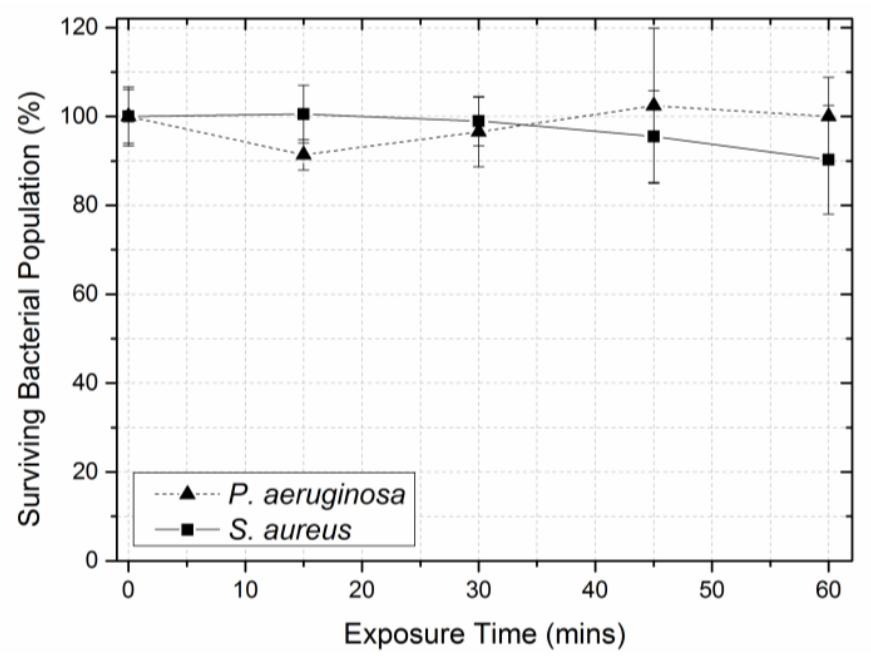

Figure 7: Inactivation of $S$. aureus and P. aeruginosa by exposure to the pulsed $R Y G$-mix. Surviving bacterial populations are calculated as a percentage of the equivalent non-exposed control population $( \pm \mathrm{SD})$. Statistical analysis showed no significant change in bacterial populations following light exposure, as compared to the equivalent non-exposed populations $(\mathrm{P}=>0.05)$.

The results show no significant difference between the exposed bacterial samples and non-exposed controls over the duration of all exposures. This demonstrates that there is no antimicrobial effect from the RYG mix. This light output induced no negative (nor positive) effects on the bacterial populations, thus demonstrating that all inactivation achieved from the blended source is achieved by the photodynamic action of the $405 \mathrm{~nm}$ light, and addition of the RYG mix can be used purely to assist in the aesthetics associated with the light output.

\section{DISCUSSION \& CONCLUSION}

This study has demonstrated that a blended white light containing pulsed $405 \mathrm{~nm}$ light can inactivate 2 common hospital acquired infections, $S$. aureus and $P$. aeruginosa showing a 3- $\log _{10}$ reduction in population. Also demonstrated was the improvement in the quality of light output in terms of CRI, with the addition of the red, yellow and green to the $405 \mathrm{~nm}$ light creating a more comfortable blend of light, aesthetically speaking.

The study by Gillespie et $a l^{13}$ showed that $405 \mathrm{~nm}$ light can be pulsed at a lower duty cycle and thus the average intensity will be lower and will appear less bright than the peak intensity whilst maintaining the same levels of inactivation. This consequently suggests that by using pulsed $405 \mathrm{~nm}$ light, the level of $405 \mathrm{~nm}$ light should appear lower whilst maintaining the inactivation achieved with continuous $405 \mathrm{~nm}$ light, thus making it easier to blend with supplementary colours in order to produce a blended white light.

In addition, practically speaking, power requirement will be a key considering in the viability of any blended white prototype. Therefore in theory, if the LEDs are pulsed on and off, then the electrical energy consumption should be lower than that of a continuous blended light system. This is also demonstrated in the study by Gillespie $e a^{13}$. Future work could investigate the optimal duty cycle and pulsed operation for most efficient bacterial inactivation in terms of electrical energy.

An additional consideration is the spread of wavelengths of light across the spectrum. The white LED spectrum shown in Figure 3 has a contiguous spread from green through to red, which is theoretically what is required to blend white light. Ideally, more LEDs across of a variety of wavelengths would have been used to create a fuller spectrum closer to a white blend but this isn't practical, so it was decided on four LEDs spread across the spectrum.

If scaled up and further developed it is possible that a white light could be developed which could replace standard white light in certain environments whilst providing the benefit of long-term environmental disinfection. Further work is 
required in order to get to this stage, looking at power consumption, and how scaling up to a useful level of light and specifically and useful level of $405 \mathrm{~nm}$ light i.e. bacterial inactivation, would effect the power requirements and size. In addition, investigation into the use of diffusers or frosted glass is required to mask the individual coloured LEDs when looking up at the light, so as to create what would appear as a large white source opposed to many different coloured individual LEDs.

The study concludes that a pulsed blended white light prototype with antimicrobial properties could be a viable solution for continuous environmental decontamination applications. The results showed that, the addition of supplementary pulsed red, yellow and green light does not affect, either negatively or positively, the antimicrobial properties of the pulsed $405 \mathrm{~nm}$ light. This means the aesthetics of the lights can be improved by the addition of the supplementary colours without any interference to the environmental decontamination objective.

\section{ACKNOWLEDGEMENTS}

JBG wishes to thank the Engineering and Physical Sciences Research Council (EPSRC) for their support through a Doctoral Training Grant (EP/L505080/1; EP/K503174/1). All authors wish to thank The Robertson Trust for their support and the High Voltage Technologies (HVT) mechanical and electronics workshop staff for their technical assistance.

\section{REFERENCES}

[1] Maclean, M., MacGregor, S. J., Anderson, J. G. and Woolsey, G., "High-intensity narrow-spectrum light inactivation and wavelength sensitivity of Staphylococcus aureus." FEMS Microbiol. Lett. 285(2), 227232(2008).

[2] Maclean, M., Murdoch, L. E., MacGregor, S. J. and Anderson, J.G., "Sporicidal effects of high-intensity 405 nm visible light on endospore-forming bacteria." Photochem. Photobiol. 89(1), 120-126(2013).

[3] Maclean, M., MacGregor, S. J., Anderson, J. G. and Woolsey, G., "Inactivation of bacterial pathogens following exposure to light from a 405-nanometer light-emitting diode array.” Appl. Environ. Microbiol. 75(7), 1932-1937(2009).

[4] Luksiene, Z., Gudelis, V., Buchovec, I. and Raudeliuniene, J., "Advanced high-power pulsed light device to decontaminate food from pathogens: effects on Salmonella typhimurium viability in vitro." J. Appl. Microbiol. 103(5), 1545-1552(2007).

[5] Enwemeka, C. S., Williams, D., Hollosi, S., Yens, D. and Enwemeka, S. K.,. "Visible 405 nm SLD light photodestroys methicillin-resistant Staphylococcus aureus (MRSA) in vitro." Lasers Surg. Med. 40(10), 734737(2008).

[6] Tomb, R. M., Maclean, M., Herron, P. R., Hoskisson, P. A., MacGregor, S. J. and Anderson, J. G., "Inactivation of Streptomyces phage $\phi$ C31 by $405 \mathrm{~nm}$ light. Requirement for exogenous photosensitizers?" Bacteriophage. 1, 1-6(2014).

[7] Maclean, M., Booth, M. G., Anderson, J.G., MacGregor, S. J., Woolsey, G. A., Coia, J. E., Hamilton, K. and Gettinby, G., "Continuous decontamination of an intensive care isolation room during patient occupancy using 405 nm light technology.” Journal of Infection Prevention 14(5), 176-181(2013).

[8] Maclean, M., MacGregor, S. J., Anderson, J. G., Woolsey, G. A., Coia, J. E., Hamilton, K., Taggart, I., Watson, S. B., Thakker, B. and Gettinby, G., "Environmental decontamination of a hospital isolation room using highintensity narrow-spectrum light.” J. Hosp. Infect. 76(3), 247-251(2010).

[9] Kowalski, W., [Ultraviolet germicidal irradiance handbook: UVGI for air and surface disinfection], SpringerVerlag, Berlin \& Heidelberg, 17-46 (2009).

[10]Dai, T., Tegos, G. P., Zhiyentayev, T., Mylonakis, E. and Hamblin, M.R., "Photodynamic Therapy for Methicillin-Resistant Staphylococcus aureus Infection in a Mouse Skin Abrasion Model." Lasers Surg Med. 42(1), 1-14(2010).

[11]Dai, T., Gupta, A., Huang, Y. Y., Yin, R., Murray, C. K., Vrahas, M. S., Sherwood, M. E., Tegos, G. P. and Hamblin, M. R., "Blue light rescues mice from potentially fatal pseudomonas aeruginosa burn infection: Efficacy, safety, and mechanism of action." Antimicrob Agents Chemother. 57(3), 1238-1245(2013). 
[12] McDonald, R. S., Gupta, S., Maclean, M., Ramakrishnan, P., Anderson, J. G., MacGregor, S. J., Meek, R. M. D. and Grant, M. H., "405 nm Light exposure of osteoblasts and inactivation of bacterial isolates from arthroplasty patients: potential for new disinfection applications?” Eur. Cell. Mater. 25, 204-214(2013).

[13] Gillespie, J. B., Maclean, M., Given, M. J., Wilson, M. P., Judd, M. D., Timoshkin, I. V. and MacGregor, S. J., "Efficacy of Pulsed 405-nm Light-Emitting Diodes for Antimicrobial Photodynamic Inactivation: Effects of Intensity, Frequency, and Duty Cycle." Photomed Laser Surg. In Press (2016).

[14] Nave, R., "Rayleigh Scattering" Hyperphysics, http://hyperphysics.phy-astr.gsu.edu/hbase/atmos/blusky.html (15th December 2016) 